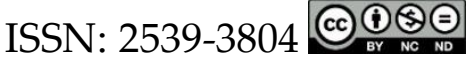

\title{
Interacción y comprensión: la reformulación docente como estrategia de interacción verbal en la universidad ${ }^{1}$
}

María Victoria Bruno Zenklusen

Universidad Nacional de Córdoba

Córdoba, Argentina

\section{Resumen}

Este artículo presenta el planteo general de una investigación que tuvo como objetivo conocer las estrategias de interacción verbal utilizadas por una docente universitaria para facilitar el aprendizaje a partir de los textos en una carrera de Ciencias Sociales. El estudio, de carácter exploratorio-descriptivo y metodología cualitativa, se efectuó en una materia de primer año de una universidad privada de la ciudad de Córdoba, Argentina. La recolección de los datos se realizó mediante el registro en audio de los intercambios verbales entre los participantes. La interpretación de los datos sigue un microanálisis discursivo centrado en las acciones de la docente, y se exponen los resultados vinculados con el uso de la estrategia de interacción verbal de re-expresar. Se observó que la profesora, cuando reformula los aportes de los estudiantes, promueve la precisión conceptual al utilizar terminología específica de la disciplina y proporciona estrategias de producción oral características de la comunidad académica.

Palabras clave: interacción verbal; estrategias de interacción; enseñanza y aprendizaje; comprensión; universidad; nivel superior.

\footnotetext{
1 Este trabajo se inscribe en el marco de una investigación concluida realizada durante el período de marzo de 2016 a agosto de 2018. Fue parte del Trabajo Final de la autora para concluir sus estudios de la Licenciatura en Español Lengua Materna y Lengua Extranjera, de la Facultad de Lenguas, Universidad Nacional de Córdoba, dirigido por la Dra. Bibiana Amado. El título de la tesis es "Interactuar para aprender: Estrategias discursivas de interacción verbal en el aula de nivel superior".
} 


\begin{abstract}
Interaction and Comprehension: Teacher Reformulation as a Verbal Interaction Strategy at University

This paper presents an overview of a research aimed at studying the verbal interaction strategies used by a university teacher to promote learning through texts during her classes in a program in Social Sciences. The study, which has a descriptive and exploratory approach and a qualitative methodology, was carried out in a first-year course at a private university in the city of Córdoba, Argentina. The primary data source were the audio recordings of verbal exchanges among participants. The interpretation of data follows a discourse microanalysis focused on the teacher's actions, and results reflect upon the use of the interactional strategy known as revoicing. It was noted that, when the teacher revoices students' contributions, she fosters conceptual precision through the use of specific discipline-related terminology and provides speaking skills that are characteristic of the academic community.
\end{abstract}

Key words: verbal interaction; interactional strategies; teaching and learning; reading comprehension; university; higher education.

\title{
Résumé
}

Interaction et compréhension : la reformulation des enseignants comme stratégie d'interaction verbale à l'université

Cet article présente les idées générales d'une recherche visant à connaître les stratégies d'interaction verbale utilisées par une professeure universitaire afin de favoriser l'apprentissage par le biais des textes dans le cadre d'une filière d'études en sciences sociales. Cette étude exploratoire-descriptif et de méthodologie qualitative a été réalisée lors des cours d'une chaire de la première année d'une université privée de la ville de Cordoba, Argentine. La collecte des données a été faite par l'enregistrement audio des échanges verbaux entre les participants. L'interprétation des données s'est basé sur une microanalyse du discours portée sur les actions de l'enseignante. Dans ce travail, on présente les résultats concernant la stratégie de l'interaction verbale de la ré-expression. Lors de la reformulation des apports des étudiants, on observe que la professeure favorise la précision conceptuelle en utilisant la terminologie spécifique de la discipline et en facilitant des stratégies de production orale caractéristiques de la communauté académique. Cette étude montre la manière dont l'enseignante facilite la compréhension d'un texte en favorisant la précision conceptuelle.

Mots-clés : interaction verbale; stratégie d'interaction; enseignement et apprentissage ; compréhension ; université ; enseignement supérieur. 


\section{CóMO CITAR ESTE ARTÍ́CULO}

Bruno Zenklusen, M. (2020). Interacción y comprensión: la reformulación docente como estrategia de interacción verbal en la universidad. Lenguaje, 48(1), 60-83. doi: 10.25100/lenguaje.v48i1.7332 


\section{INTRODUCCIÓN}

En los distintos niveles educativos, el aprendizaje a partir de los textos requiere del acompañamiento de lectores expertos que tengan dominio del tema que se aborda y que, a su vez, puedan promover habilidades de comprensión. Los desarrollos de la psicología cognitiva sobre los modelos de comprensión de textos sugieren que en este proceso es fundamental que el lector haya logrado comprender de manera profunda el contenido textual mediante la integración de esa información con su conocimiento previo (Kintsch, 1994). Los aportes desde este campo permiten conocer los procesos cognitivos que entran en juego en todo proceso de lectura, las habilidades requeridas para lograr un buen desempeño y, en ocasiones, las maneras más adecuadas de realizar la tarea.

Diferentes investigaciones en el nivel superior, universitario y no universitario, plantean que el desempeño en términos de lectura y escritura por parte de los estudiantes, en general, no se ajusta a las expectativas de ese nivel. Así, las indagaciones acerca de la enseñanza y del aprendizaje en esos ámbitos educativos han cobrado importancia en las últimas décadas. Estas, por un lado, arrojan conclusiones que permiten describir las características de los procesos y las prácticas de lectura y escritura, y las dificultades que manifiestan los estudiantes en la comprensión y producción de textos académicos (Arnoux, Nogueira y Silvestri, 2002; De Arnoux, Nogueira y Silvestri, 2006; Di Stefano y Pereira, 1997, 2004, 2009, 2012, 2013). Por otro lado, los estudios desde la corriente de alfabetización académica (Carlino, 2003, 2006) resultan ser muy útiles al momento de la descripción del fenómeno, asumiendo que la cuestión es una responsabilidad compartida por todos los miembros de la comunidad académica y, puntualmente, de los docentes de las distintas materias. Estos deben "hacerse cargo de la lectura y la escritura" (Carlino, 2001) para que los estudiantes aprendan las habilidades necesarias para reconstruir los saberes disciplinarios de cada campo.

Todos estos trabajos son muy productivos, debido a que muestran una situación problemática y brindan herramientas a nivel descriptivo para abordarla. No obstante, se encuentran limitaciones importantes. Por una parte, todos estos enfoques se limitan a presentar el estado de situación en relación con la lectura y la escritura en la universidad para, finalmente, centrar su atención en esta última. Por otra, no abundan análisis de las prácticas de enseñanza, en concreto, de las interacciones verbales entre docentes y estudiantes que mediatizan dichos procesos.

La perspectiva desarrollada por Di Stefano y Pereira (1997, 2004, 2009, 2012, 2013), por ejemplo, considera la lectura y la escritura como procesos cognitivos, prácticas sociales y prácticas discursivas, aunque, luego, focaliza fuertemente en este último aspecto. Desde un enfoque sociodiscursivo y centrados en la noción de representación social, presentan distintas características de la lectura y la escritura en la universidad, muy diferentes de otros contextos y niveles educativos, como la escuela. En este sentido, estas investigadoras sostienen que los actores involucrados, sobre todo en los primeros años, llevan a cabo el habitus correspondiente a una cultura 
institucional y discursiva diferente, más acorde con las exigencias de un contexto escolar, que no se ajusta al contexto universitario (Di Stefano y Pereira, 2012).

Los resultados de sus estudios, efectuados principalmente en el marco de los talleres de lectura y escritura del Ciclo Básico Común de la Universidad de Buenos Aires, presentan ciertos rasgos de las representaciones sociales que tienen los estudiantes sobre la lectura. Entre ellos, puede mencionarse la lectura obediente y descontextualizada, orientada a la retención de datos, con escasa o nula relación con los conocimientos previos sobre un tema, entre otros. Estos rasgos se tornan relevantes, debido a que estas representaciones sobre la tarea de lectura de un texto teórico en el ámbito académico inciden en distintas instancias de la lectura. Entre ellas, las autoras destacan la construcción del marco de lectura, la jerarquización de la información, el establecimiento de relaciones entre fuentes y de relaciones causales y el control de la propia comprensión (Di Stefano y Pereira, 1997).

Por ende, el ingreso a la universidad requiere de nuevos aprendizajes en relación con las prácticas de lectura y escritura propias de la comunidad académica. En otras palabras, este "pasaje" (Di Stefano y Pereira, 2004) de una cultura institucional a otra, tal como lo conciben, prevé la adopción de nuevas prácticas, de otras formas de leer y de escribir. Estos desafíos suponen transformaciones profundas en el nivel de las representaciones sociales que los estudiantes tienen en el momento de ingresar acerca de esos procesos y, también, de las tareas necesarias para tener un buen desempeño.

Estos estudios ponderan el trabajo en talleres de lectura y escritura, y brindan herramientas didácticas para diagnosticar las dificultades y facilitar esos aprendizajes. Sus conclusiones resaltan los beneficios de trabajar estas falencias iniciales en dichos espacios de taller. En sus comunicaciones, estas autoras destacan que en la "puesta en común oral" (Di Stefano y Pereira, 1997, p. 336) con los docentes de los talleres, los estudiantes manifestaron tener conocimientos previos sobre las temáticas que debían desarrollar en sus producciones, pero tuvieron claras dificultades en sus respuestas escritas. Sin embargo, sus análisis no ahondan en esos momentos de discusión oral y, por ello, destacamos estas anotaciones sobre la puesta en común en la que se producen interacciones entre docentes y estudiantes, y las ponderamos como espacios clave para los procesos de enseñanza y de aprendizaje en la universidad.

De manera semejante, Riffo y Contreras (2012) defienden la idea de que ser estudiante universitario se corresponde con cierta competencia mínima respecto del uso de la lengua formal culta y que la formación apunta, entre otras cuestiones, a mejorarla. En cada disciplina, el discurso especializado es un pilar fundamental (Parodi, 2005) y los miembros de la comunidad académica deben aprender a manejarlo para pertenecer al campo. Así, estos autores plantean que el nivel de formación académica y el grado de especialización del discurso inciden en el desempeño de los estudiantes para comprender textos académicos. De allí las diferencias encontradas entre alumnos de primer año en relación con los de años más avanzados, por lo que es de esperar que quienes se inician en su formación no cuenten 
con las herramientas indispensables para comprender textos complejos y específicos $\mathrm{y}$, por lo tanto, deban aprenderlas.

Por su parte, Lizardo (2006) sostiene que en la universidad deben dirigirse los esfuerzos por llevar a cabo prácticas educativas estructuradas a partir de los estudiantes y "que privilegien el desarrollo de intercambios verbales en el interior del aula de clase" (p. 673). En esas circunstancias, la atención a estos aspectos implica que los docentes consideren a los estudiantes sujetos activos de su formación y asuman su rol docente para propiciar esos espacios de coproducción. Desde este marco, esta autora analiza el patrón de interacción verbal en el aula universitaria para indagar los procesos cognitivos promovidos en los estudiantes.

Para su investigación, utiliza la metodología propuesta por Flanders, desarrollada en la década de 1970 (González y León, 2009; Lizardo, 2006; Mercer y Dawes, 2014) y concebida como un modo de analizar la interacción a través de dos patrones, uno de influencia directa y otro de influencia indirecta. Estos patrones están integrados por diferentes categorías que, luego, permiten codificar las acciones de los docentes y, de esta manera, asignarles un valor numérico. En esas categorías se tienen en cuenta factores como el nivel de control en el manejo de las actividades, la estimulación a la participación, la elaboración de preguntas que estimulen la reflexión, entre otros. De acuerdo con esa metodología, el patrón de influencia indirecta establece la figura docente como aquella persona que orienta su práctica hacia situaciones que promueven el desarrollo de habilidades y estrategias cognitivas de los estudiantes (Lizardo, 2006), a la vez que busca y permite su participación activa. Por su parte y contrariamente, el patrón de influencia directa presenta un profesor guiado por criterios autoritarios y de control, que limita la participación.

Entre sus resultados, postula que, más allá de que la docente participa en un número mucho mayor de intervenciones, esta maneja un patrón de interacción de influencia indirecta. Lizardo (2006) afirma que la exposición docente es una actividad que se sostiene como una de las características principales de la enseñanza en la universidad. Sin embargo, se advierte un claro interés de su parte por incorporar a los estudiantes en su formación profesional y como sujetos activos en la construcción del conocimiento y la expresión de sus puntos de vista sobre los contenidos.

Una experiencia semejante es la desarrollada por Martínez-Díaz, Díaz y Rodríguez (2011), que combina el enfoque sociocultural del aprendizaje con el modelo interactivo de comprensión lectora elaborado por Van Dijk y Kintsch (1983). Esta investigación hace una indagación de un programa de andamiaje asistido (Wood, Bruner y Ross, 1976) para mejorar la comprensión lectora en estudiantes universitarios. En ella, analizan el rol de los docentes como lectores expertos en el proceso de formación y buscan diseñar e implementar un programa de andamiaje. Sin embargo, los profesores de su estudio no consideran que deban tener una participación activa en la comprensión lectora de los alumnos como parte de su rol docente. En este punto, no asumen estas acciones como una función central de su tarea universitaria, ya que creen que son habilidades que los estudiantes deben tener aprendidas. 
Como puede observarse, la reflexión sobre los procesos y las prácticas de enseñanza y de aprendizaje en la universidad es vasta y variada. Sin embargo, no abundan los casos que estudien los modos en que estos se promueven por medio de situaciones interactivas y en las que los docentes operen como facilitadores de estrategias de comprensión. De esta manera, la interacción verbal en el aula de nivel superior entre docentes y estudiantes aparece como un tema clave para la investigación, ya que con sus resultados pueden aportarse más herramientas para abordar estos fenómenos. Es por ello que interesa indagar acerca de lo que sucede en las clases en la universidad para conocer qué hacen los docentes para facilitar la lectura y la comprensión de textos de los estudiantes en carreras de Ciencias Sociales.

\section{MARCO TEÓRICO Y EMPÍRICO}

La enseñanza y el aprendizaje en la universidad y su relación con los procesos lingüísticos y cognitivos necesarios para un buen desempeño y aquellos que pueden ser promovidos en instancias de interacción verbal requieren un abordaje desde un punto de vista que asuma la complejidad. Por esta razón, considerar la lectura no solo en su aspecto cognitivo, sino también como una práctica social y discursiva (Di Stefano y Pereira, 1997, 2004) cobra especial relevancia para situar la problemática. Teniendo en cuenta este enfoque, el presente trabajo se plantea desde una perspectiva que combina los aportes de la teoría socio-histórico cultural del aprendizaje (Bruner, 1984; Vygotski, 1978) y los estudios de interacción verbal en el aula (Borzone, 2005; Mercer y Dawes, 2014; O'Connor y Michaels, 1993, 1996).

\section{La teoría socio-histórico cultural y el aprendizaje en la universidad}

Desde la perspectiva socio-histórico cultural del desarrollo humano propuesta por Vygotski (1978), se entiende que el aprendizaje se logra en y a través de la interacción con sujetos más expertos. En este planteo, el concepto de zona de desarrollo próximo (ZDP) refiere a la distancia entre el nivel de desarrollo real y el potencial. El primero se relaciona con la capacidad de una persona para realizar una tarea de manera autónoma, mientras que el segundo se determina por la resolución de algún problema con la guía de alguien más experto. En este proceso, el lenguaje es una herramienta semiótica fundamental, principalmente para el desarrollo de los procesos psicológicos superiores. Estos, entre los que se incluye la comprensión del lenguaje escrito, requieren de instancias de socialización específicas (Martínez-Díaz et al., 2011; Silvestri, 2000b) y, como consecuencia, el rol que asumen los más expertos es clave. En este sentido, Vygotski (1978) plantea que el aprendizaje primero es social, esto es, a nivel interpersonal, y, luego individual, cuando se internaliza.

En esta línea, la noción de andamiaje (Bruner, 1984; Wood et al., 1976), elaborada como aplicación pedagógica del concepto de ZDP (Martínez-Díaz et al., 2011), alude a un sistema de apoyo al aprendizaje que se realiza en la interacción. Así, se concibe que los más expertos, en nuestro caso, los docentes, promueven la puesta en marcha de razonamientos y acciones que facilitan el proceso para que los novatos 
se apropien de los saberes expertos. Estos aportes acerca de cómo aprendemos representan las bases del planteo de la investigación presentada en este artículo para estudiar la interacción verbal en la universidad.

Partiendo de estas bases teóricas, el trabajo de Silvestri (2000b) sobre el enfoque sociocultural y los aportes acerca de los efectos cognitivos de los géneros conceptuales en el ámbito escolar (Silvestri, 2000a) plantean ciertas líneas interpretativas que pueden ser consideradas, en términos generales, para la descripción de los discursos del ámbito académico, entre los que ubicamos el nivel superior, tanto universitario como no universitario. De acuerdo con su presentación, el ámbito académico se constituye por aquellas prácticas institucionales y discursivas que involucran la docencia y la investigación, en sus diversos géneros y dispositivos, ya sean orales o escritos. Estas prácticas sociales construyen el conocimiento y determinan las maneras de producirlo y comunicarlo (Silvestri, 2000b), como también cómo y dónde se llevan a cabo.

Ahora bien, los géneros discursivos no solo tienen funciones comunicativas que son específicas de cada uno, sino que, además, mediatizan procesos cognitivos (Silvestri, 2000b; Wertsch, 1993). En otras palabras, la forma y el contenido se encuentran estrechamente vinculados en la comprensión del discurso. Entonces las prácticas de enseñanza que tienen lugar en, por ejemplo una clase universitaria, promueven procesos cognitivos. Así, las interacciones entre docentes y estudiantes en actividades de puesta en común ponen en relación los aportes de cada participante, los conocimientos previos y los contenidos de los textos. En este contexto, la información compartida se caracteriza, como otros tipos de géneros académicos, por el uso de un léxico específico y una sintaxis compleja, pese a ser oral. Consecuentemente, para su comprensión es necesario conocer las particularidades del género y realizar "determinadas operaciones mentales tales como, por ejemplo, en el caso del texto teórico, la elaboración de una red conceptual jerárquica perteneciente a un dominio de conocimiento o la identificación del sustento conceptual abstracto que subyace en una práctica" (De Arnoux et al., 2006, p. 142).

Dicho esto, en el ámbito de la educación superior, los estudiantes ingresan en una comunidad discursiva que tiene un dominio de conocimiento específico (Riffo y Contreras, 2012) y, ante esta situación, deben aprender saberes ya institucionalizados que son productos configurados culturalmente a lo largo de la historia. En otras palabras, las formas de construcción del conocimiento, de producción y de comunicación de dichos saberes están preestablecidos. Como consecuencia, los nuevos agentes en el campo (Bourdieu, 2003), en este caso los estudiantes de primer año de la universidad, deben aprender las prácticas que caracterizan a dicha comunidad (Di Stefano y Pereira, 1997). Entonces, el ingreso y la permanencia en una institución educativa de nivel superior requieren la adopción de nuevas herramientas cualitativamente distintas de las utilizadas para cumplir las expectativas de otros niveles educativos.

Sin embargo, en estos contextos, la heterogeneidad de saberes y habilidades de las personas que concurren a la universidad son aspectos evidentes (De Arnoux et al., 
2006) $\mathrm{y}$, en ocasiones, el desempeño de los estudiantes no siempre está en concordancia con las exigencias del nivel (Carlino, 2003; Di Stefano y Pereira, 1997). Los textos involucrados en los aprendizajes de este sistema tienen un alto grado de complejidad, vinculado con los procesos de abstracción propios de cada ciencia y, en su mayoría, son textos teóricos. Entre sus principales características pueden mencionarse la presencia de secuencias expositivo-explicativas y argumentativas con elementos variados, tanto desde la estructura gramatical como los fenómenos discursivos, que suelen presentar dificultades para la comprensión. Así, se destacan una gran densidad léxica y una densidad verbal baja, procedimientos de referencia intra e intertextuales complejos, entre otras (Bronckart, 1996; De Arnoux et al., 2006). Ante esto, la acción docente debe atender al hecho de que los estudiantes, sobre todo en el primer año, se encuentran ante el desafío de reconstruir "una red conceptual jerárquica perteneciente a un dominio de conocimiento o la identificación del sustento conceptual abstracto que subyace en una práctica" (De Arnoux et al., 2006, p. 142) que, para muchos, es novedosa.

Siguiendo a Campbell (1988), una de las pocas maneras con las que contamos para acceder a lo que los estudiantes comprendieron a partir de la lectura de los textos es a través de lo que pueden verbalizar, ya sea oralmente o por escrito. Es por esto que cobra relevancia la combinación del enfoque sociocultural del aprendizaje con las consideraciones de Silvestri (2000a, 2000b) sobre los géneros discursivos y los estudios sobre la lectura y la escritura en la universidad para destacar la importancia del estudio de la interacción verbal y su relación con los procesos de enseñanza y aprendizaje en este ámbito educativo.

\section{Los estudios de la interacción en el aula}

Una indagación de la bibliografía sobre la enseñanza y el aprendizaje desde un enfoque interaccional permite recuperar diversas formas de estudiar dichos procesos. A partir de la década del setenta, surgieron numerosas investigaciones acerca de lo que sucedía en las aulas en términos de adquisición del lenguaje en niños de habla inglesa. Los primeros trabajos al respecto, que se llevaron a cabo en el Reino Unido y los Estados Unidos, atendían a la actividad docente, y entre sus aportes más destacados se encuentran su propuesta de la estructura que tomaban las interacciones en las aulas (Mehan, 1979; Sinclair y Coulthard, 1975). Posteriormente, se iniciaron distintas líneas de investigación desde perspectivas que articulaban, en su mayoría, el enfoque socio-histórico cultural del aprendizaje y sus desarrollos posteriores (Bruner, 1984; Nelson, 1996; Rogoff, 1993), con los postulados teóricos y metodológicos provenientes de otras disciplinas, como la sociología, la antropología y la psicología cognitiva. Estos estudios permitieron el desarrollo del campo científico de la interacción en el aula y su relación con los procesos de enseñanza y de aprendizaje.

Estas combinaciones construyeron distintos objetos. Algunos estudiosos los abordaron teniendo en cuenta el punto de vista del texto social y las relaciones sociales, mientras otros se centraron en la construcción del texto académico (Mercer y 
Dawes, 2014). En el presente trabajo, interesa la perspectiva que centra su atención en este segundo punto, es decir, "en el proceso colaborativo que da lugar a la construcción conjunta de significados y a la comprensión de nuevos contenidos" (Borzone, 2005, p. 122). En esta dinámica, las funciones de las estrategias de interacción verbal utilizadas por los docentes tienen un rol muy importante en el proceso de aprendizaje y en la construcción conjunta del conocimiento.

Por su parte, O'Connor y Michaels $(1993,1996)$ hacen sus estudios en escuelas primarias de Estados Unidos aplicando una metodología cualitativa de microanálisis de la interacción en el aula de dos maestras con vasta experiencia en el uso de estrategias de esta índole en sus clases de matemáticas y ciencias. Estas autoras definen un tipo particular de acción que denominan revoicing, que luego Borzone (2005) recupera con la traducción de re-expresar. En la presente comunicación, se utilizan estos términos, o reformular, ya que en los antecedentes se los utiliza de manera indistinta y no constituyen una contradicción con el marco teórico asumido. Esta estrategia refiere a la acción de los docentes en la interacción cuando retoman las contribuciones de un estudiante y las reelaboran (O'Connor y Michaels, 1996). Estas reelaboraciones o este parafraseo pueden realizarse con un cambio a nivel proposicional o en el léxico utilizado. Asimismo, las investigadoras plantean el uso del discurso indirecto por parte de los docentes a través de verbos de decir y verbos de pensamiento, con los que los estudiantes se ven interpelados como los enunciadores de dicha reformulación, más allá de que haya sido el/la docente quien la formuló. Otra forma lingüística recurrente es el uso de marcadores discursivos para indicar aquellas reformulaciones que buscan una reelaboración de inferencias en función de lo sucedido en turnos anteriores. Tal es el caso de "entonces", que los docentes usan, por lo general, al comienzo de sus turnos de habla para aceptar y reexpresar la contribución anterior, y agregar nueva terminología.

En este sentido, O'Connor y Michaels (1996) marcan una diferencia con la tradicional secuencia IRE (iniciación-respuesta-evaluación) (Cazden, 1988; Mehan, 1979; Sinclair y Coulthard, 1975) o las formulaciones posteriores como secuencia IRF (movimiento follow-up) (Mercer y Dawes, 2014). De acuerdo con su crítica hacia estas propuestas, los docentes, con el último movimiento de la secuencia, se reservan el derecho a evaluar la contribución de los estudiantes y no dan la posibilidad de negociar el significado. En cambio, sostendrán estas autoras, mediante el revoicing, los docentes aceptan las respuestas de los estudiantes, usan sus contribuciones como base para la (re)elaboración de inferencias y dan a los alumnos la posibilidad de ratificarlas. De este modo, se genera un cambio en la responsabilidad o autoría de los enunciados y se produce una negociación del significado y una construcción en conjunto del texto académico. A su vez, proponen que los formatos de participación que dinamiza la estrategia de re-expresar posibilitan demostrar la relación entre los movimientos interaccionales y el modo en que las ideas y las prácticas de pensamiento grupal son construidas conjuntamente.

En la Argentina, un número considerable de investigaciones adoptaron la interacción verbal y no verbal como matriz social de todo aprendizaje (Borzone, 2005; 
Rosemberg, Borzone y Diuk, 2003), esto es, como mediadora de los procesos de enseñanza y de aprendizaje. Concretamente, cuando hablamos de estrategias de interacción verbal nos remitimos a los trabajos de Rosemberg (2002), Rosemberg et al. (2003) y Borzone (2005). Estas investigadoras promueven la articulación de los estudios sobre la interacción verbal en el aula con los postulados de la psicología cognitiva en lo referido a la comprensión y la producción, desde un enfoque sociohistórico cultural del aprendizaje. El presente artículo se inscribe en esta línea de investigación y considera la categoría revoicing descripta previamente como un antecedente directo de la estrategia de re-expresar.

Dichos trabajos hacen hincapié en la interacción docentes-niños en situaciones de lectura de diferentes tipos de textos. Entre sus conclusiones sostienen que, trabajando en ZDP, esas interacciones, además de ser un sistema de apoyo a la comprensión de un texto concreto, promueven el desarrollo de estrategias de comprensión en los niños (Borzone, 2005). En otras palabras, durante estas situaciones, las docentes ponen a disposición habilidades de lectoras expertas al momento de la lectura y operan como memoria extendida del grupo (Borzone, 2005). Se espera que esas acciones que resuelven con la ayuda de las docentes puedan hacerlas solos. Esto se logra cuando se produce, en términos de Vygotski (1978), la internalización de esas funciones cognitivas, y se realiza un aprendizaje efectivo. Sin embargo, este punto es difícil de conocer y excede los objetivos de estos trabajos, así como el de esta investigación.

En esta línea, la interacción entre los participantes de una clase de nivel superior constituye una instancia propicia para la construcción conjunta de un texto oral a través de las distintas contribuciones (Borzone, 2005). Aunque estas situaciones pueden ser más o menos planificadas, un rasgo característico es la contingencia. En este proceso, los docentes guían esa interacción mediante acciones, que aquí se denominan estrategias de interacción verbal, para favorecer ciertos intercambios e inhibir otros, de acuerdo con el objetivo didáctico perseguido en cada ocasión. En este contexto áulico, los docentes que fomentan la participación deben prestar atención a las reacciones de los estudiantes para construir el texto académico deseado. Sin embargo, no deja de ser una relación asimétrica que se manifiesta en la palabra de los docentes como parte autorizada para controlar y distribuir la palabra y, a la vez, ser quienes determinan la adecuación o inadecuación de una contribución.

Hecho este planteo general, el presente artículo sostiene que la mediación docente en la interacción facilitaría los procesos de comprensión de los estudiantes universitarios. Los docentes como lectores expertos (Martínez-Díaz et al., 2011) situarían "la información en el marco de ideas y procedimientos claves que estructuran el pensamiento en el seno del ámbito disciplinar concreto" (Elliott, citado en De Arnoux et al., 2006, p. 161). Esta tarea requiere, necesariamente, la cooperación de los estudiantes que aporten con sus contribuciones para coproducir el texto académico oral a partir de la lectura previa de un texto. Se considera que la estrategia de interacción de re-expresar puede facilitar estos aprendizajes a partir de la 
promoción de la precisión conceptual. Esas reformulaciones funcionan como andamiaje (Bruner, 1984; Wood et al., 1976) desplegado en ZDP (Vygotski, 1978). En este sentido, puede pensarse en una mente social, operando en una zona de desarrollo colectiva (Hernández, 1999). Estas acciones de los docentes son fundamentales para ese "pasaje" (Di Stefano y Pereira, 1997) hacia la comunidad discursiva de la universidad, para que se internalicen los nuevos contenidos.

\section{Metodología}

\section{Participantes y justificación del caso}

Este trabajo se enmarca en una investigación más amplia de carácter exploratoriodescriptivo que se llevó a cabo desde un enfoque cualitativo. El trabajo de campo se realizó en una institución universitaria privada de la ciudad de Córdoba, Argentina, en el año académico 2016, en una materia de primer año de las Licenciaturas en Ciencia Política y en Relaciones Internacionales. La elección de este caso se justifica por un criterio de oportunidad, puesto que los datos se recabaron durante el primer año de adscripción de la autora en su carácter de egresada de la Licenciatura en Ciencia Política de dicha unidad académica. Además, se contó con la generosidad, el interés y la autorización de toda la cátedra para llevar a cabo la investigación.

Por otro lado, se considera un caso paradigmático, debido a la dinámica propuesta por la cátedra para el desarrollo de los temas. Su relevancia radica en que durante las clases se promueve activamente la participación de los estudiantes, que es fundamental para el funcionamiento de la clase. Además, es una materia en la que se estudian temas relacionados con los paradigmas de las Ciencias Sociales que requieren el desarrollo de una reflexión crítica sobre la ciencia, la tarea de investigación y el rol de los estudiantes como futuros productores de conocimiento. Los textos obligatorios estipulados por el programa plantean una gran complejidad y algunas dificultades para su comprensión, según comentó la docente titular de ese año en una entrevista informal previa a la recolección de los datos.

La información empírica utilizada corresponde al registro de una clase en la que participaron la profesora titular y 45 estudiantes que, al momento del registro de los datos, cursaban la materia. El corpus se conformó por situaciones de interacción verbal entre los participantes y para su elaboración se siguió un criterio de exhaustividad, ya que se seleccionaron aquellos intercambios que proporcionaran elementos para conceptualizar y caracterizar la estrategia de re-expresar. Para ello, se priorizaron aquellas instancias en las que se observó su uso para explorar su relación con la comprensión. El presente artículo recorta esos resultados y se centra en la estrategia de re-expresar y en la manera en que esta promueve la precisión conceptual.

\section{Procedimientos de recolección y de análisis de datos}

El procedimiento utilizado para recopilar los datos fue la observación de clases con registro anecdótico de los intercambios entre docente y estudiantes, esto es, se trabajó 
con actividades no programadas ni controladas por la investigadora. El registro se obtuvo por medio de la grabación digital de audio, contando con la autorización previa y el consentimiento de los participantes. Luego, se procedió a la transcripción ortográfica.

Con relación a la transcripción ortográfica, dada la calidad del audio, algunos estudiantes no pudieron ser identificados y se decidió señalar sus voces con la letra "E". En los otros casos, los nombres fueron modificados para preservar su identidad. Los turnos de habla correspondientes a la docente se señalan mediante la letra " $\mathrm{D}$ ". Los puntos suspensivos entre corchetes marcan un recorte de las intervenciones que se apartan de los contenidos planificados y cuyo recorte no genera distorsiones para su interpretación. Este recurso se utiliza debido a que el objetivo es identificar las partes de los intercambios que evidencian el uso de la estrategia de re-expresar. Por su parte, el guion medio señala autointerrupciones en el momento de hablar, o bien, interrupciones a los coparticipantes. Entre paréntesis se presentan la duración en segundos de las pausas intraturnos. Además, los segmentos incomprensibles fueron puestos entre paréntesis con un espacio en blanco. Para finalizar estas aclaraciones, los comentarios de la investigadora se ponen entre doble paréntesis.

Una vez finalizada esta tarea, se segmentaron las interacciones teniendo en cuenta las herramientas conceptuales provistas por el análisis conversacional, en lo referido a la estructura, a saber, turnos, intercambios y secuencias (Sacks, Schegloff y Jefferson, 1974). Los turnos se separan a partir de las intervenciones de cada interactuante $\mathrm{y}$, al producirse su alternancia entre diferentes participantes, las emisiones son interpretadas como intercambios. Estos, a su vez, pueden ser agrupados en secuencias cuando la interacción presenta una estructura interna que remite a un mismo tópico. De esta forma, a partir de la división en secuencias, se reconocieron los temas de la clase, las características de los inicios y cierres de las secuencias, y los mecanismos de regulación de la interacción. Estos conceptos fueron de gran utilidad al momento de describir las situaciones de interacción.

A continuación, se realizó un microanálisis discursivo de las interacciones (Borzone, 2005; Gumperz y Field, 1995). Para complementar, se siguieron algunas consideraciones metodológicas tomadas de Edwards y Mercer (1988) y Campbell (1988).

El objetivo de este análisis fue reconocer las estrategias de interacción verbal de la docente que promovieran procesos de comprensión. Las categorías que guiaron esta tarea analítica se obtuvieron de trabajos previos. De la investigación de Borzone (2005), se recuperaron las estrategias retomar, expandir, solicitar expansión y reexpresar. Si bien en la descripción de los intercambios seleccionados para este artículo intervienen todas ellas, se tomó especial consideración por la estrategia de re-expresar. Esta es la traducción recuperada del trabajo de Borzone (2005), en referencia directa a la propuesta de revoicing de O'Connor y Michaels $(1993,1996)$. También se refiere a ella como reformulación. 
Una vez reconocidos los intercambios en los que se utilizó esta estrategia, se hizo una descripción de los enunciados de cada interlocutor prestando especial atención a lo realizado por la docente para interpretar la funcionalidad de dicho movimiento en cada situación concreta. La pregunta guía de esta operación analítica fue: ¿Qué está haciendo cuando utiliza esta estrategia y para qué? (Campbell, 1988). La finalidad de esta pregunta era indagar sobre los procesos de comprensión que la estrategia de re-expresar estaba (o no) facilitando.

\section{RESULTADOS Y DISCUSIÓN}

El análisis de los datos ha permitido reconocer distintas situaciones en las que la docente utiliza la estrategia de re-expresar y promueve la precisión conceptual mediante su uso. Antes de abordar dichos intercambios, es preciso compartir ciertas consideraciones generales del contexto de la clase trabajada. La observación de diferentes clases mostró que, durante el desarrollo de los temas, se producen situaciones de interacción entre las docentes de la cátedra y los estudiantes de manera constante, promovidas en su mayoría por iniciativa de la profesora.

Teniendo en cuenta los registros anecdóticos efectuados y las entrevistas previas con el equipo docente, puede decirse que las docentes conciben la enseñanza y el aprendizaje como procesos interactivos y asumen un rol activo que no se limita a exponer los temas a partir de clases magistrales. Asimismo, consideran que, a través de la puesta en común de las lecturas y el debate sobre estos y los materiales trabajados, se genera una construcción conjunta del conocimiento en el que es muy importante la participación de los estudiantes en la dinámica para que se desarrollen los contenidos de la clase.

De acuerdo con lo que explicitó la docente, con esos intercambios tiene como objetivo propiciar la reflexión acerca de los modos de producción y de validación del conocimiento científico, como también de su futuro rol como profesionales y como productores de conocimiento. De la misma manera, expresó que, dada su experiencia, el texto trabajado en la clase estudiada suele presentar muchas dificultades, ya que se caracteriza por una alta densidad léxica, sintagmas extensos, dimensión polémica, entre otros rasgos, complejidad que queda registrada en las interacciones. Por esta razón, la profesora busca que los estudiantes intervengan y hagan aportes significativos en las clases para que se involucren en su propio proceso de formación y construyan entre todos los temas objeto de estudio. Además, considera que con esas interacciones se contribuye al desarrollo de estrategias propias de la comunidad discursiva académica, sobre todo a la lectura crítica (Di Stefano y Pereira, 2009) y a la reflexión sobre los temas, los textos, a su rol de estudiantes y demás aspectos de la vida académica. A decir de Di Stefano y Pereira (1997), intenta transformar sus representaciones sociales en torno a dichas prácticas y procesos.

Así, la dinámica de las clases tiene como eje central incentivar la participación. Tal como destacan Martínez-Díaz et al. (2011), estos procesos de apoyo como los que se plantean en este trabajo solo son posibles con el involucramiento de los estudiantes. 
Para esto, se exige una lectura cuidadosa y el estudio previo de los textos. En este contexto, los estudiantes que formaron parte de la investigación muestran un gran compromiso, puesto que se involucran y participan en la dinámica propuesta y contribuyen con sus turnos al entramado interaccional que la docente espera en cada clase. Ello se manifiesta en la cantidad de intervenciones, a pesar de que siempre participan entre diez y quince estudiantes, aproximadamente. No obstante esa cantidad, la docente siempre se dirige al grupo en general y busca la participación de todos.

\section{La interacción verbal como mediadora en la comprensión de textos}

A partir del análisis del corpus elicitado, se han identificado, conceptualizado y caracterizado distintas estrategias presentes en las interacciones entre la docente y los estudiantes. Como resultados del estudio, solo a efectos ilustrativos debido al alcance del presente artículo, se observó que la docente retoma y expande, o bien, retoma y solicita expansión, simplemente solicita expansión, re-expresa (o reformula) lo que dicen los estudiantes y re-estructura cuando toma aportes de varios alumnos y hace una exposición más larga de algún tema o concepto. En relación con los procesos cognitivos que promovían esas estrategias durante la interacción, se interpretaron los siguientes procesos, a saber, la promoción de habilidades metacognitivas, la activación de conocimiento previo, el desarrollo de procesos inferenciales, la organización y jerarquización de la información y la promoción, de precisión conceptual.

Para este trabajo, se tuvo en cuenta la categoría re-expresar como estrategia de interacción verbal para promover la precisión conceptual. Con esta acción, la docente retoma los enunciados proferidos por un estudiante y los reelabora o reformula, ya sea para clarificar o remarcar aspectos de las contribuciones de los alumnos. De esta manera, elimina algunos vocablos, usa diferentes palabras a las expresadas por su interlocutor y, en ocasiones, agrega nueva información. Esta acción permite reelaborar y reconceptualizar lo dicho por un estudiante en su emisión con términos más específicos. Todo ello se interpreta con un valor estratégico en relación con el contenido académico que se desea desarrollar. En este sentido, la reformulación posibilita clarificar y ampliar aquellas ideas, muchas veces expresadas en un lenguaje informal, e introducir nueva terminología. Así, esta estrategia promueve el desarrollo de la producción oral acorde a la comunidad académica. Un intercambio significativo en el que se identifica esta situación es el siguiente:

D: ¡Alterando el orden! Rompiendo el orden, al estilo marxista, ¿sí? ¿Qué le molestaba a Comte del espíritu de la Ilustración?

Luis²: El caos que generaba.

\footnotetext{
${ }^{2}$ Los nombres de los estudiantes fueron modificados para resguardar su identidad. Se contó con el consentimiendo de los participantes de la clase para grabar y utilizar los datos recolectados.
} 
D: Su carácter revolucionario. El problema con el ideario de la Ilustración no tenía que ver con sus principios y supuestos, sino con su- casi, con su metodología. [...]

Otro aspecto, de acuerdo con O'Connor y Michaels (1996), es que, mediante la reformulación, los docentes aceptan las respuestas de los estudiantes y las utilizan como base para la elaboración de inferencias y, en ocasiones, dan a los alumnos el derecho de ratificarlas. De esta forma, se genera un cambio en la responsabilidad o autoría del contenido que se hace público, lo que permite una negociación del significado y una construcción conjunta del tema de la clase. Además, con este movimiento, la docente aporta coherencia a un texto académico oral, en el cual la voz de los estudiantes se vuelve imprescindible. Tal es el caso del ejemplo que se presenta a continuación:

D: ¿Qué más? (0.5) ¿Qué pasa con esos estadios? Vamos a la ley de los estadios, de los tres estadios. ¿Qué pasa con los estadios, con el metafísico y con el teológico, según Comte?

Juan: Dice que, con el avance del conocimiento y de la sociedad, con el tiempo, como que el teológico y el metafísico van quedando viejos y, en realidad, el único que, digamos, en su actualidad, digamos, el único válido sería el positivo, el estadio positivo.

D: ¿Estaríamos de acuerdo en decir que, según la teorización de Comte, los estadios teológicos y metafísicos fueron superados por el estadio positivista? Juan: Por lo que yo comprendí, sí.

D: ¿El resto?

$[\ldots]$

A través de esta investigación se propone que la estrategia de re-expresar de la docente en la interacción verbal con los estudiantes forma parte de un proceso de andamiaje (Bruner, 1984) al aprendizaje, es decir, de un sistema de apoyo a la comprensión como una actividad conjunta que opera en ZDP (Vygotski, 1978). En este proceso, la docente proporciona estrategias de comprensión que pone a disposición de los estudiantes para promover el aprendizaje a partir del texto y orienta el desarrollo de diferentes procesos cognitivos. Entre ellos se destaca la promoción de la precisión conceptual que se detallará a continuación.

\section{La reformulación docente como estrategia de interacción verbal para promover la precisión conceptual}

Como se mencionó, el objetivo de promover la precisión conceptual se lleva a cabo a través de distintas estrategias. Sin embargo, interesa destacar el rol de la reformulación en esta tarea. En la situación propuesta anteriormente, se observa el uso 
de la estrategia de re-expresar, que pone a disposición de los estudiantes herramientas conceptuales y léxicas correspondientes al discurso académico.

En el ejemplo anterior, la docente, en un primer momento, utiliza la estrategia de solicitar expansión para avanzar en el desarrollo del tema. Esto puede interpretarse en la emisión “¿Qué pasa con esos estadios? Vamos a la ley de los estadios, de los tres estadios. ¿Qué pasa con los estadios, con el metafísico y con el teológico, según Comte?". La repetición del enunciado interrogativo se reconoce como una forma de dirigir la atención del grupo hacia la nueva información solicitada. La docente enuncia una consigna oral que repite para dar precisión a su pregunta, lo cual se observa en la mención de los estadios a los que se refiere, para ubicar el tema que quiere abordar. En este caso, la guía directa pauta los contenidos que se hacen públicos en la interacción y la repetición presenta la claridad en la formulación de las ideas como aspecto clave. Ante esta acción, un estudiante contribuye con una respuesta breve y satisfactoria a la que la docente reacciona con una intervención más extensa en comparación con las que venía produciendo. Este movimiento se identifica como estrategia de re-expresar.

Por medio de una reformulación con características del discurso académico, la docente incluye la voz del estudiante y promueve procesos de producción oral. Los recursos lingüísticos utilizados son señales de esta promoción, al expresar mediante un registro formal y un léxico más preciso la idea compartida por el estudiante. Se reconocen dos ejemplos paradigmáticos en este punto. En primer lugar, re-expresa el verbo decir realizado por el alumno en tercera persona del singular, tiempo presente del modo indicativo, "dice", cuya referencia resulta ambigua, dado que puede aludir a Comte como autor del que están hablando o al texto trabajado como fuente de su aporte. Teniendo en cuenta la reacción de la profesora, podemos afirmar que dicha ambigüedad fue reconocida por la docente, ya que reformula el enunciado proporcionando la precisión propia del discurso académico: "según la teorización de Comte". Así, contribuye a construir el significado en un plano más abstracto, producto de la negociación, puesto que es el resultado de ambas contribuciones.

Otro ejemplo se encuentra en el segundo turno de la profesora cuando dice "fueron superados", señal de reelaboración de lo dicho por el estudiante en el enunciado "van quedando viejos". En ambos casos, la docente reconoce como pertinentes las respuestas dadas por Juan en sus turnos de habla y con el uso de la estrategia posiciona al estudiante como la fuente última para la convalidación de la reformulación realizada. Dicho de otro modo, el objetivo de parafrasear la emisión tiende a incluirlo para deliberar el significado último de lo que quiso decir con su contribución. La forma lingüística elegida para plantear la reformulación es una interrogación introducida por "estaríamos de acuerdo" en tiempo condicional y el uso del nosotros inclusivo.

Estas acciones de la profesora evidencian no solo una reelaboración, sino que, además, manifiesta, en términos del análisis conversacional (Sacks et al., 1974), una búsqueda de convergencia entre los participantes, es decir, llegar a un acuerdo con el 
estudiante que hizo el aporte. Cuando la docente re-expresa y expande, logra retomar un turno de habla e introducir un resumen que une sus emisiones con lo dicho anteriormente por Juan. A partir de allí, el tono ascendente presente en la pregunta señala el pase para que el estudiante pueda ratificar la inferencia que aporta la docente, hecho que realiza de la siguiente forma: "Por lo que yo comprendí, Sí". Teniendo en cuenta la resolución final del conflicto cognitivo presentado por la docente que se da minutos más tarde, el razonamiento expuesto por Juan es correcto. Sin embargo, la profesora no evalúa explícitamente su respuesta, sino que inicia otro turno con una pregunta dirigida al resto de la clase para que aporten nuevas ideas.

Algo similar ocurre en el siguiente caso, en el que la docente reelabora y completa la pregunta formulada por un estudiante. Estos movimientos se identifican con la estrategia de re-expresar, que permite un avance en el desarrollo del contenido de la clase. En esta línea, O’Connor y Michaels (1996) plantean que la docente asume su rol de moderadora de la clase y transmite a la audiencia el enunciado propuesto por algún estudiante. Al realizar esta acción, utiliza un léxico más preciso y, al mismo tiempo, hace partícipe al resto del grupo con una pregunta que, en un principio, fue dirigida a ella. Desde una perspectiva cognitiva, esta intervención implica la extensión de un conflicto cognitivo a todo el grupo iniciado, en primera instancia, con la duda de un estudiante.

\section{Sergi: Profe, ( ～） ((Emisión reconocida como una pregunta))}

D: Una persona con un posicionamiento político reformista nunca apoyaría un sistema social, político y de conocimiento basado en fundamento- Silencio, chicos, porque hay ruido afuera, ustedes hacen ruido acá, me hacen gritar muy mucho. Si hablamos, lo hacemos para todos. Estoy explicándole algo al compañero. ¿Escucharon lo que preguntó?

Flavia: Sí.

D: ¿Qué preguntó?

Flavia: Que no entendió por qué la posición de Comte era reformista.

D: Sí, vos estás al lado. El fondo, ¿escuchó lo que él preguntó? O sea, ¿yo pa' qué me gasto explicando una cosa que es importante política y epistémicamente, si la mitad de la clase no sabe de qué estamos hablando? Volvé a hacer tu pregunta.

E: Bueno, yo decía que no entendí bien que la posición de Comte es reformista, si a los monárquicos...

[...]

D: No, no. Yo te entiendo, quiero que sea una pregunta para todos, para todos. Un reformista, ¿cómo convive con un sistema que basa su principio de autoridad en Dios o en el derecho natural divino, o en el derecho natural y divino, o sea, una abstracción filosófica?

La duda planteada por este alumno es presentada en términos fragmentarios $\mathrm{y}$, de hecho, en voz muy baja. La docente reformula y completa la pregunta para ser 
retransmitida a todos los presentes en la clase. Asimismo, estos intercambios se interpretan como parte del habla de control y un modo de llamar la atención del grupo, puesto que muchos estudiantes no prestan atención a la pregunta de su compañero. Así, la profesora inhibe acciones no expertas en una instancia de puesta en común o debate ante las dudas de un compañero. Por medio de esta acción, problematiza las representaciones de sí mismos como estudiantes universitarios (Di Stefano y Pereira, 1997), ya que la estrategia de re-expresar incluye la voz del estudiante y le otorga responsabilidad en la reformulación.

Mediante esta elección, la docente sitúa el aporte en un nivel de abstracción mayor, puesto que re-expresa con terminología precisa. Tales son los casos de "sistema", "principio de autoridad", "derecho natural", "derecho divino", "abstracción filosófica", conceptos que tienen significados específicos en las Ciencias Sociales, según la perspectiva que se asuma, con lo cual los estudiantes deben aprender este aspecto del discurso científico y académico para comprender y producir enunciados pertinentes. De esta forma, la reformulación promueve el aprendizaje de elementos de producción oral con la introducción de léxico específico de mayor abstracción.

Como puede observarse, el análisis de la estrategia de interacción de reexpresar de la docente que participó de esta investigación permite reconocer la relevancia de la intervención tanto en un plano comunicativo como en un plano cognitivo. Desde la perspectiva sociocultural, ambas dimensiones han sido señaladas como aspectos fundamentales del lenguaje (Silvestri, 1998). En concreto, se tornan relevantes el uso de estas categorías para estudiar la interacción verbal en el aula y los procesos y las prácticas de enseñanza y de aprendizaje en el nivel superior. Los intercambios que pueden tener lugar en el aula constituyen un apoyo y contribuyen significativamente a que los estudiantes aborden temas y textos complejos, y desarrollen habilidades de lectores expertos (Martínez-Díaz et al., 2011) en una nueva comunidad discursiva. Con el análisis de la estrategia de re-expresar se encontró que, durante el proceso de interacción, la profesora promueve el desarrollo de habilidades cognitivas para la comprensión de conceptos científicos propios del campo de las Ciencias Sociales. En este caso, se focalizó en la promoción de la precisión conceptual y se observó que la docente, a través del uso de la estrategia de interacción verbal de re-expresar, aporta terminología específica de la disciplina y brinda elementos del discurso académico.

\section{CONCLUSIONES}

Este artículo presenta el planteo teórico-metodológico de una investigación más amplia que considera como andamiaje positivo las estrategias de interacción verbal de los docentes en las clases de nivel universitario para promover el aprendizaje a partir de los textos. Se hizo hincapié en la estrategia de re-expresar, categoría recuperada de estudios previos (Borzone, 2005; O'Connor y Michaels, 1993). Esta se refiere al movimiento discursivo realizado por la docente cuando reformula los aportes de los 
estudiantes y la función principal de esta acción, en relación con el desarrollo de estrategias de comprensión, es la promoción de la precisión conceptual.

Al reelaborar los enunciados de los alumnos, la profesora introduce nueva terminología y brinda herramientas del discurso expositivo-explicativo típico de las prácticas de la comunidad académica. En este contexto donde las dificultades son evidentes, la docente modeliza la producción de un texto oral coherente mediante un registro formal y un léxico más preciso. En esta misma línea, facilita la construcción de significados más abstractos, siempre a partir de los aportes de los estudiantes. En este aspecto, la negociación de los significados es indispensable para la continuación de la interacción. Mediante estas acciones, la docente problematiza las representaciones de los estudiantes universitarios (Di Stefano y Pereira, 1997), puesto que incluye su voz y les otorga responsabilidad en la reformulación.

Asimismo, se observaron situaciones en las que la docente reelabora preguntas con un léxico más preciso y, en lugar de responder directamente, redirige la pregunta al resto de la clase. Así, involucra a todo el grupo en la búsqueda de respuestas (Campbell, 1988) y se dedica a moderar la clase. En este sentido, las situaciones observadas evidencian que tanto la guía de la docente a través de la estrategia de reexpresar como la participación activa de los estudiantes resultan clave para la construcción del texto oral y el desarrollo de los contenidos pautados en el programa de la asignatura.

La combinación de la perspectiva socio-histórico cultural del aprendizaje y el enfoque interaccional permite interpretar la comprensión como proceso conjunto, que se construye en una zona de desarrollo colectiva (Hernández, 1999). La estrategia de re-expresar constituye un sistema de apoyo a la comprensión que opera en ZDP (Vygotski, 1978). En este proceso de andamiaje (Bruner, 1984) al aprendizaje, estos intercambios favorecen y orientan el desarrollo de diferentes procesos cognitivos, esto es, la comprensión y la producción de conceptos más abstractos correspondientes a la lógica de la disciplina. Del mismo modo, se trata de recuperar el potencial del encuentro y concebir las interacciones en las clases como espacios propicios para la transformación de las representaciones sociales acerca de la lectura y la escritura que tienen los estudiantes en su etapa inicial de formación (Di Stefano y Pereira, 1997).

En otro orden, el registro anecdótico, la grabación en audio y su posterior transcripción ortográfica han permitido reconstruir los intercambios de manera fiel a lo acontecido, a pesar de ciertas dificultades, como la mala calidad del audio en algunos momentos. La adopción de un enfoque cualitativo brinda la flexibilidad en el diseño de la investigación, sin por ello prescindir de la exhaustividad y rigurosidad del estudio. Las distintas decisiones teórico-metodológicas contribuyeron a un abordaje satisfactorio para el conocimiento de las estrategias de interacción verbal utilizadas por la docente en sus clases. Por esta razón, los resultados de este estudio no pueden ni pretenden ser generalizados a otras situaciones. Sin embargo, puede considerarse un antecedente importante para estudiar casos similares y para conocer los modos en que puede facilitarse el aprendizaje a partir de los textos en el nivel superior. 
El estudio de lo que ocurre efectivamente en las clases es un campo abierto y prometedor para la investigación. Para ello, es indispensable conocer las formas que asumen, en la actualidad, los procesos y las prácticas de enseñanza y de aprendizaje en los distintos ámbitos educativos y, concretamente, en la universidad. Esta investigación propone recuperar categorías y técnicas de investigación generalmente olvidadas al momento de abordar prácticas educativas. Un aspecto relevante en este tipo de análisis es la ampliación de la semiosis que permiten los avances tecnológicos aplicados a las técnicas de recolección de datos. El enfoque interaccional brinda herramientas teórico-metodológicas muy valiosas para investigar estos fenómenos, teniendo en cuenta otras modalidades que van más allá de la dimensión verbal para dar sentido a dichas prácticas.

\section{REFERENCIAS}

Arnoux, E., Nogueira, S., y Silvestri, A. (2002). La construcción de representaciones enunciativas: el reconocimiento de voces en la comprensión de textos polifónicos. Signos, 35(51-52), 129-148. doi: 10.4067/S0718-09342002005100010.

Borzone, A. (2005). Conversar y Comprender: El Desarrollo de Estrategias de Comprensión a través del Discurso Compartido. Revista Mexicana de Psicología, 22(1), 121-135. Recuperado de http://www.redalyc.org/pdf/2430/243020643012.pdf.

Bourdieu, P. (2003). El oficio del científico. Barcelona: Anagrama.

Bronckart, J.-P. (1996). Activité langagière, textes et discours. Pour un interactionisme socio-discursif. París: Delachaux et Niestlé.

Bruner, J. (1984). Acción, Pensamiento y Lenguaje. Madrid: Alianza Editorial.

Campbell, D. R. (1988). Desarrollo de la alfabetización en matemáticas en una clase bilingüe. En J. Cook-Gumperz (Ed.), La construcción social de la alfabetización (pp. 179-211). Barcelona: Paidós.

Carlino, P. (2001). Hacerse cargo de la lectura y la escritura en la enseñanza universitaria de las ciencias sociales y humanas. I Jornadas sobre La lectura y la escritura como prácticas académicas universitarias, Universidad Nacional de Luján, Provincia de Buenos Aires. Recuperado de https://www.aacademica.org/paula.carlino/178.

Carlino, P. (2003). Leer textos científicos y académicos en la educación superior: Obstáculos y bienvenidas a una cultura nueva. Uni-Pluri/Versidad, 3(2), 1-9. Recuperado de http://aprendeenlinea.udea.edu.co/revistas/index.php/unip/article/view/12289.

Carlino, P. (2006). Ayudar a leer en los primeros años de universidad o de cómo convertir una asignatura en «materia de cabecera». Educación en Ciencias, 1-12. Recuperado de https://www.aacademica.org/paula.carlino/158.

Cazden, C. B. (1988). Classroom Discourse. The Language of Teaching and Learning. Portsmouth: Heinemann. 
De Arnoux, E., Nogueira, S., y Silvestri, A. (2006). Lecturas y reescrituras de un texto teórico en estudiantes de profesorado de enseñanza primaria. Signo y Seña, (16), 137-165. doi: 10.34096/sys.n16.5712.

Di Stefano, M., y Pereira, M. (1997). Representaciones sociales en el proceso de lectura. Signo y Seña, (8), 319-340. doi: 10.34096\%2Fsys.n8.5783.

Di Stefano, M., y Pereira, M. (2004). La enseñanza de la lectura y la escritura en el nivel superior: procesos, prácticas y representaciones sociales. En P. Carlino (Coord.), Textos en Contexto $\mathrm{N}^{\circ}$ 6. La lectura y la escritura en la Universidad (pp. 23-39). Buenos Aires: Lectura y Vida/Asociación Internacional de Lectura.

Di Stefano, M., y Pereira, M. (2009). Modernidad y posmodernidad en discursos sobre la lectura en el ámbito educativo (2001-2006). En M. Pini (Coord.), Discursos y educación. Herramientas para el análisis crítico (pp. 233-262). Buenos Aires: UNSAM.

Di Stefano, M., y Pereira, M. (2012). Concepción sociodiscursiva de la lectura y la escritura y su enseñanza. Presentado en el IV Congreso Internacional de Investigación y Práctica Profesional en Psicología, Buenos Aires, Argentina. Recuperado de http://semiologia-cbc-distefano.com.ar/publicaciones/articulos/diStefano-Pereira-Ponencia-IV-Congreso-Psicologia.pdf.

Di Stefano, M., y Pereira, M. (2013). La formación de lectores en la educación superior: la lectura de concepciones teóricas implícitas en los debates sociales. Presentado en el II Congreso Internacional de Profesores de Lenguas Oficiales del MERCOSUR, Ciudad Autónoma de Buenos Aires, Argentina.

Edwards, D., y Mercer, N. (1988). El conocimiento compartido: El desarrollo de la comprensión en el aula. Barcelona: Paidós.

González, B., y León, A. (2009). Interacción verbal y socialización cognitiva en el aula de clase. Acción Pedagógica, 18(1), 30-41. Recuperado de http://www.saber.ula.ve/handle/123456789/29540.

Gumperz, J., y Field, M. (1995). Children's discourse and inferential practices in cooperative learning. Discourse Processes, 19(1), 133-147. doi: 10.1080/01638539109544908.

Hernández, G. (1999). La zona de desarrollo próximo. Comentarios en torno a su uso en los contextos escolares. Perfiles Educativos, 21(85-86), 46-71. Recuperado de http://www.iisue.unam.mx/perfiles/numeros/1999/85-86.

Kintsch, W. (1994). Text comprehension, memory, and learning. American Psychologist, 49(4), 294-303. doi: 10.1037/0003-066X.49.4.294.

Lizardo, S. (2006). Los procesos cognitivos y el patrón de interacción verbal en el aula universitaria. Educere, 10(35), 671-678. Recuperado de http://www.saber.ula.ve/handle/123456789/20123.

Martínez-Díaz, E., Díaz, N., y Rodríguez, D. E. (2011). El andamiaje asistido en procesos de comprensión lectora en universitarios. Educación y Educadores, 14(3), 531-556. doi: 10.5294/edu.2011.14.3.5.

Mehan, H. (1979). Learning Lessons: Social Organization in the Classroom. Cambridge: Harvard University Press. 
Mercer, N., y Dawes, L. (2014). The study of talk between teachers and students, from the 1970s until the 2010s. Oxford Review of Education, 40(4), 430-445. doi: 10.1080/03054985.2014.934087.

Nelson, K. (1996). Language in Cognitive Development: The Emergence of the Mediated Mind. Cambridge: Cambridge University Press.

O'Connor, M., y Michaels, S. (1993). Aligning Academic Task and Participation Status through Revoicing: Analysis of a Classroom Discourse Strategy. Anthropology and Education Quarterly, 24(4), 318-335. doi: 10.1525/aeq.1993.24.4.04x0063k.

O'Connor, M., y Michaels, S. (1996). Shifting participant frameworks: Orchestrating thinking practices in group discussion. En D. Hicks (Ed.), Discourse, learning, and schooling (pp. 63-103). Cambridge: Cambridge University Press.

Parodi, G. (2005). La comprensión del discurso especializado escrito en ámbitos técnico-profesionales: ¿Aprendiendo a partir del texto? Revista Signos, 38(58), 221-267. doi: 10.4067/S0718-09342005000200005.

Riffo, B., y Contreras, M. (2012). Experiencia académica y comprensión de textos especializados en estudiantes universitarios de Ciencias Políticas. Estudios Pedagógicos, 38(2), 201-219. Recuperado de http://mingaonline.uach.cl/pdf/estped/v38n2/art13.pdf.

Rogoff, B. (1993). Aprendices del pensamiento. El desarrollo cognitivo en el contexto social. Barcelona: Paidós.

Rosemberg, C. (2002). La conversación en el aula: un contexto para el aprendizaje. Una revisión de las investigaciones sobre el tema. Lingüística en el aula, (5), 7-28.

Rosemberg, C., Borzone, A., y Diuk, B. (2003). La lectura de textos expositivos: Estrategias de interacción para acercar los textos a los niños. Interdisciplinaria, 20(2), 121-145. Recuperado de https://www.redalyc.org/pdf/180/18020201.pdf.

Sacks, H., Schegloff, E. A., y Jefferson, G. (1974). A Simplest Systematics for the Organization of Turn-taking in Conversation. Language, 50(4), 696-735. doi: 10.2307/412243.

Silvestri, A. (1998). En otras palabras: habilidades de reformulación en la producción del texto escrito. Buenos Aires: Cántaro.

Silvestri, A. (2000a). Los géneros discursivos escolares: un aprendizaje discursivo y cognitivo. En Lenguajes: Teorías y prácticas (pp. 187-192) Buenos Aires: Secretaría de Educación.

Silvestri, A. (2000b). Los géneros discursivos y el desarrollo del pensamiento. Un enfoque sociocultural. En S. Dubrovsky (Comp.), Vigotski: Su proyección en el pensamiento actual (pp. 87-95). Buenos Aires: Novedades Educativas.

Sinclair, J., y Coulthard, M. (1975). Towards an Analysis of Discourse: The English Used by Teachers and Pupils. Londres: Oxford University Press.

Van Dijk, T., y Kintsch, W. (1983). Strategies of Discourse Comprehension. Nueva York: Academic Press.

Vygotski, L. (1978). El desarrollo de los procesos psicológicos superiores. Barcelona: Grijalbo. 
Wertsch, J. (1993). Voces de la mente: Un enfoque sociocultural para el estudio de la acción mediada. Madrid: Visor.

Wood, D., Bruner, J., y Ross, G. (1976). The Role of Tutoring in Problem Solving. Journal of Child Psychology and Psychiatry, 17(2), 89-100. doi: 10.1111/j.14697610.1976.tb00381.x.

\section{SOBRE LA AUTORA}

\section{María Victoria Bruno Zenklusen}

Licenciada en Español Lengua Materna y Lengua Extranjera por la Facultad de Lenguas, Universidad Nacional de Córdoba y Licenciada en Ciencia Política por la Universidad Católica de Córdoba. Integrante del Proyecto de Investigación "Plurilingüismo y educación: prácticas, representaciones, enseñanza y aprendizaje de lenguas" (FL-UNC). Áreas de interés académico: enfoque interaccional, didáctica de la lectura y la escritura en la Universidad, sociolingüística, glotopolítica, políticas lingüísticas, diversidad y derechos lingüísticos.

Correo electrónico: vicky.bruno89@gmail.com

Orcid: 0000-0002-3009-1121. 